\title{
Proactive interference in sentence recall: Topic-similarity effects and individual differences
}

\author{
FRANK N. DEMPSTER \\ University of Nevada, Las Vegas, Nevada
}

\begin{abstract}
The effect of topic similarity on proactive interference in sentence recall was investigated in two experiments using the Brown-Peterson paradigm. In Experiment 1, successive sentences about the same topic and related topics were both associated with substantial and progressive amounts of proactive interference; however, a significant trials $\times$ condition interaction indicated that the effect was stronger in the related-topics condition. Moreover, significant negative correlations were found between individual differences in susceptibility to proactive interference and academic achievement test scores. In Experiment 2, relatively weak effects leveling off on the second trial were obtained with sentences about unrelated topics. With some assumptions about the spread of activation during retrieval, the results were attributed to response-set interference. The practical implications of these data were also considered, and it was suggested that proactive interference might be a common cause of learning problems.
\end{abstract}

Proactive interference has been of central interest to students of learning and memory since the influential work of Underwood (1957). Historically, this interest has focused largely on simple verbal material, such as unrelated nouns, and it is now abundantly clear that this material is highly susceptible to proactive interference, particularly when successive units are similar. In the widely used Brown-Peterson task, for example, the presentation of items from the same taxonomic category (e.g., animal) for three consecutive trials results in a large and progressive decrease in correctly recalled words (see Wickens, 1972).

But what of proactive interference in memory for connected discourse, and to what extent is proactive interference a factor in everyday learning? Studies pertinent to this question fall into one of two groups, depending on the materials used. In one group are studies in which the conditions of learning were rather atypical of everyday learning conditions. In one such study, interference was found when the same set of characters were engaged in different activities and relationships in two different stories (Thorndyke, 1977). In another, interference occurred when the specific details (e.g., names, dates, places) of interpolated passages differed from those in the target passage (Thorndyke \& Hayes-Roth, 1979). For example, interpolated sentences such as "This constellation was originally charted at Mount Wilson observatory, " in-

This research was supported by grants from the Dean of the College of Education and the University of Nevada, Las Vegas. Research Council. I thank Felecia M. Briscoe. Jeri O'Bannon-Plunkett. and Bryan K. Scott for their assistance. Requests for reprints should be sent to Frank N. Dempster. College of Education. University of Nevada. Las Vegas. NV 89154 terfered with memory for changed arguments in sentences such as "This constellation was originally charted at Palomar Observatory." Although a student is sometimes taught a multiple listing of arguments to the same predicates, this is likely to constitute only a small portion of what a student is asked to learn. Accordingly, these two studies show that meaningful material is susceptible to proactive interference, but they fail to show that proactive interference is an important factor in everyday learning.

In the second group are studies that used more naturalistic materials. In these, subjects who read passages (Blumenthal \& Robbins, 1977) or read and listened to television news items (Gunter, Berry, \& Clifford, 1981; Gunter, Clifford, \& Berry, 1980) about the same topic (e.g. physics) showed a significant decline in learning across trials, whereas subjects who read topically different units exhibited no decline at all. Moreover, the interference accumulated rapidly and had a substantial effect on performance. Although these studies suggest that proactive interference may play an important role in everyday memory, their educational significance is limited by the fact that topic similarity was broadly defined and unevenly distributed across units. In the sequence about physics, for example. one passage dealt with the relative nature of time and space, another with the motion of a body, a third with the measurement of an object's mass, and a fourth with a phenomenon called "creep." Hence, the precise sense in which the passages were topically similar is not clear. What is needed now are more analytic studies in which the conditions of similarity are varied and more carefully controlled.

The chief aim of this study was to assess the effects of two forms of topic similarity (same topic and related topics: Experiment 1) and an unrelated-topics condition 
(Experiment 2) on the recall of successively presented sentences. Sentences, rather than passages, were used, in an effort to provide more control over topic similarity than seemed possible with passages. A second aim of this study was to examine the relationship between individual differences in susceptibility to proactive interference and measures of academic achievement. This issue is also of practical importance, inasmuch as a significant relationship should obtain if proactive interference is an important factor in memory for connected discourse. To this end, American College Test (ACT) scores were obtained for subjects for whom such data was available.

\section{EXPERIMENT 1}

\section{Method}

Subjects. The subjects were 80 undergraduate students enrolled in educational psychology courses at the University of Nevada, Las Vegas. They participated on a voluntary basis for extra course credit. Students who indicated that they had taken the ACT gave the experimenter written permission to obtain their scores from the Testing and Evaluation Center on campus.

Design and Materials. The design was mixed, with similarity condition (same topic, related topics) and order (A, B) as betweensubjects factors and blocks (1-6) and trials (1-4) as within-subject factors. Each subject was assigned randomly to the two betweensubjects factors until each of the four cells had 20 subjects.

Three sentences about each of six related topics, anxiety, hostility, withdrawal, inhibition, depression, and hysteria, and one sentence each about clans, glass, Antarctica, lightning, potatoes, and mathematics were constructed by the experimenter. The sentences about personality states were constructed largely on the basis of information found in the descriptive literature accompanying the Taylor-Johnson Temperament Analysis (TJTA) Scale Profile, and to a lesser extent on information found in a dictionary. The other sentences were constructed on the basis of information found in an encyclopedia and the Mosaic, a publication of the National Science Foundation. Each sentence comprised 19 words; the first word of each sentence denoted the topic, and subsequent words described the topic. In addition, all sentences were written in a similar style and without striking variations in complexity. Thus, all sentences shared certain structural characteristics. Finally, key descriptive words were not repeated frequently, either within or across sentences. Although these control measures tended to make the sentences more homogeneous and less repetitious than they might otherwise have been, more stringent measures were not imposed because it was believed that an extremely high degree of control would produce highly artificial sentences and series of sentences.

The sentences were recorded on audio cassette tapes in a slightly slower than normal reading rate in four different arrangements representing the four combinations of the two between-subjects factors of similarity condition and order. Each arrangement comprised six blocks of four sentences, as shown in Table 1 . Several characteristics of the design should be noted. First, the sentences presented on Trial 4 always occupied the same position in every trial block. This trial served primarily as a buffer trial designed to "release" any proactive interference that had developed over Trials 1-3, and thus to help prevent the spread of proactive interference from block to block. Second, several precautions, in addition to having multiple blocks, were taken to minimize the likelihood that trial-by-trial comparisons, both within and between similarity conditions, would reflect differences in sentence difficulty. First, Order A of the sametopic condition was constructed by randomly assigning sentences to Trials 1-3. Then Order B was constructed by reversing the trial sequence of Order A. Second, within each of the orders, sentences in the related-topics condition were assigned the same trial number as in the same-topic condition.
Procedure. The subjects participated individually in a small quiet room ordinarily used for counseling purposes. Upon entering the room, the subjects listened to a tape that provided information about the task. That their tasks were to try to remember sentences and to count backward accurately was emphasized. They were instructed that they should recall as much of each sentence as possible and that it would help if they thought about the sentence's meaning. Furthermore, it was suggested that if they could not recall all the words in a sentence in order, they should try to recall as many key words or ideas as possible. After these instructions were given, the procedure (discussed below) was described.

Each trial began with the word "Ready," followed by a 2 -sec interval and then the sentence. The sentence was presented twice, with the two presentations separated by a 3-sec interval. The second presentation was followed by a 3 -sec interval and then a threedigit number (a different number was used on each trial), from which the subject counted backward by threes for $15 \mathrm{sec}$. At the conclusion of this 15-sec interval, the subject heard the word "Recall," which was followed by a 15 -sec recall interval. When it followed the first three trials in a block, "Ready," signaled the end of the recall interval and the beginning of the next trial, whereas the end of the fourth and final trial of each block was signaled by the word "Stop." For every block, except the last, "Stop" was followed by a 2 -min rest interval, during which the subject was engaged in casual conversation. The choice of a 2 -min interval was based on research that had shown that a 2 -min intertrial interval effectively prevented the spread of proactive interference from trial to trial (Hopkins, Edwards, \& Cook, 1972; Loess \& Waugh, 1967). Thus, there were two precautions taken-the first was the use of a fourth trial about an unrelated topic-to prevent the spread of proactive interference from block to block.

In addition to the instruction, two practice trials were given prior to the experiment proper. These trials were administered in the same manner as the test trials, but with five unconnected words (familiar concrete nouns) per trial, instead of sentences. Two minutes elapsed between the end of the last practice trial and the start of Block 1 .

\section{Results and Discussion}

Recall was scored in two ways: verbatim (synonyms and changes in tense were acceptable) and verbatim plus credit for paraphrases of the original material (the subjects in the related-topics condition were just as likely to recall the topic word, which changed from trial to trial, as were the subjects in the same-topic condition; thus, credit was given for recall of the topic word). For example, one subject attempting to recall Sentence 1 gave the following: "Inhibition is normally combined with selfcenteredness and lack." By verbatim standards, he received a score of 6 ("Inhibition, is, self, centeredness, and, lack"), whereas by verbatim-plus standards, he received a score of 9 (credit was given for "normally combined with," which is a paraphrase of "ordinarily accompanied by').

The verbatim-plus method was scored independently by two scorers, who seldom disagreed. Moreover, since paraphrases were highly correlated with verbatim scores $(r=.92)$, the results of only the verbatim scoring method, for which there were no disagreements, were selected for analysis.

Preliminary analysis (the alpha for all analyses was set at .05) indicated that neither order nor block was associated with systematic effects. Most importantly, there was no evidence of proactive interference across successive blocks: The overall recall scores for Blocks 1 through 
Table 1

Design and Materials of Experiment 1 (Order A Is Shown; Order B Was Generated by Reversing the Order of Sentences 1-3 in Each Block)

\begin{tabular}{|c|c|c|}
\hline $\begin{array}{c}\text { Trial } \\
\text { Number }\end{array}$ & $\begin{array}{l}\text { Sentence } \\
\text { Notation }\end{array}$ & Actual Sentence \\
\hline & & Block 1 \\
\hline 1 & $A_{1}\left(A_{1}\right)$ & Anxiety is found in those who feel basically inadequate, and who therefore fear disapproval, punishment, and loss of love. \\
\hline 2 & $A_{2}\left(W_{2}\right)$ & $\begin{array}{l}\text { Anxiety is known to be characteristic of young people under stress of academic demands, vocational indecision, or } \\
\text { family conflict. }\end{array}$ \\
\hline 3 & $\mathbf{A}_{3}\left(\mathrm{D}_{3}\right)$ & Anxiety is usually associated with a conscious or unconscious fear of embarrassment or failure in some phase of life. \\
\hline \multirow[t]{2}{*}{4} & $\mathrm{C}_{1}\left(\mathrm{C}_{1}\right)$ & Clans consist of people who believe they descend from a common ancestor who lived in the distant mythological past. \\
\hline & & Block 2 \\
\hline 1 & $\mathrm{H}_{1}\left(\mathrm{H}_{1}\right)$ & $\begin{array}{l}\text { Hostility may have a harmful, destructive effect on personal, marital, and parental relationships, since it is usually } \\
\text { severely alienating. }\end{array}$ \\
\hline 2 & $\mathrm{H}_{2}\left(\mathrm{I}_{2}\right)$ & Hostility may be expressed in angry arguments that are either too prejudiced or emotionally biased to have much validity. \\
\hline 3 & $\mathrm{H}_{3}\left(\mathrm{Hy}_{3}\right)$ & $\begin{array}{l}\text { Hostility is often associated with dominance, excessive criticism, and little tolerance for opposing points of view and } \\
\text { life-styles. }\end{array}$ \\
\hline \multirow[t]{2}{*}{4} & $\mathrm{G}_{1}\left(\mathrm{G}_{1}\right)$ & $\begin{array}{l}\text { Glass, even in its unstrengthened state, is an enormously useful material that is inexpensive, noncorrosive, and light } \\
\text { in weight. }\end{array}$ \\
\hline & & Block 3 \\
\hline 1 & $W_{1}\left(W_{1}\right)$ & $\begin{array}{l}\text { Withdrawal is found in varying degrees of intensity, ranging from cases of mild self-conscious restraint to extreme } \\
\text { isolation. }\end{array}$ \\
\hline 2 & $\mathrm{~W}_{2}\left(\mathrm{Hy}_{2}\right)$ & $\begin{array}{l}\text { Withdrawal tendencies may grow progressively worse, until the individual avoids coping with even the simplest challenges } \\
\text { of everyday life. }\end{array}$ \\
\hline 3 & $\mathrm{~W}_{3}\left(\mathrm{I}_{3}\right)$ & Withdrawal often occurs in those who consciously suffer from deep feelings of inadequacy and who tend to feel inferior. \\
\hline 4 & Ant $_{1}\left(\right.$ Ant $\left._{1}\right)$ & $\begin{array}{l}\text { Antarctica is an ice-covered land surrounded by waters that house virtually untapped sources of marine protein and } \\
\text { minerals. }\end{array}$ \\
\hline
\end{tabular}

Block 4

$1 \quad I_{1}\left(I_{1}\right) \quad$ Inhibition is ordinarily accompanied by self-centeredness and lack of concern for anyone who has different beliefs or standards.

$2 \quad I_{2}\left(A_{2}\right) \quad$ Inhibition may be conditioned by an environment that forces severe emotional repression and checks any spontaneous desire to share.

$3 \quad \mathrm{I}_{3}\left(\mathrm{H}_{3}\right) \quad$ Inhibition stems from emotional blocking in those unable to express inner feelings, for fear of being hurt or rejected.

$4 \quad L_{1}\left(L_{1}\right) \quad$ Lightning is a natural spark, miles long, which usually accompanies a thunderstorm that developed from flat-bottomed cumulus clouds.

\section{Block 5}

$1 \quad \mathrm{D}_{1}\left(\mathrm{D}_{1}\right)$

$2 \quad \mathrm{D}_{2}\left(\mathrm{H}_{2}\right)$

Depression, when it becomes unbearable, gives way to desperate escape reactions such as manic moods of self-righteous elation.

$3 \quad \mathrm{D}_{3}\left(\mathrm{~A}_{3}\right)$

Depression tends to occur in emotionally unbalanced individuals who react in an exaggerated and irrational manner to everyday hardships.

$3 \mathrm{D}_{3}\left(\mathrm{~A}_{3}\right)$

$4 \quad P_{1}\left(P_{1}\right)$ centrate.

Potatoes are vulnerable to many invading plant organisms, one of which caused a severe famine in nineteenth-century Ireland.

Block 6

$1 \quad \mathrm{Hy}_{1}\left(\mathrm{Hy}_{\mathrm{s}}\right) \quad$ Hysteria is generally characterized by repressed desires and conflicts which are either avoided or displaced onto an inanimate object.

$2 \mathrm{Hy}_{2}\left(\mathrm{D}_{2}\right) \quad$ Hysteria is typically marked by flamboyant attention-seeking behavior, often vivid and seductive, that others find phony and disagreeable.

$3 \mathrm{Hy}_{3}\left(\mathrm{~W}_{3}\right) \quad$ Hysteria may included irrational fears of disturbances in bodily functions which may result in imagined pain, disease, or paralysis.

$4 \quad M_{1}\left(M_{1}\right) \quad$ Mathematics, though often considered difficult and inapplicable, is useful in a vast range of commercial, technological, and professional occupations.

Note-The notation in parentheses indicates the order used in the related-topics condition.

6 , respectively, were $6.69,5.64,5.27,5.63,6.14$, and 5.46. Therefore, subsequent analyses were collapsed over these factors. The primary analysis design, then, was a repeated-measures ANOVA, with similarity condition a between-subjects factor and trials (1-3) a within-subject factor.

The results of this analysis revealed that the overall ef- fect of similarity condition was not significant $[\mathrm{F}(1,78)=$ 1.62], but there was a significant trials effect $[F(2,156)=$ 84.61] and a significant condition $\times$ trials interaction $[F(2,156)=5.30]$. These effects are depicted graphically in Figure 1, which presents the mean number of correct words recalled as a function of similarity condition and trials. As can be seen, recall declined substantially across 
trials in both conditions; however, it declined more steeply in the related-topics condition. Direct statistical evidence of proactive interference was provided by simple contrasts between successive trials within each condition. These contrasts revealed that the decline in performance between Trials 1 and 2 and Trials 2 and 3, respectively, was significant in both the same-topic condition $[\mathrm{F}(1,156)=$ 15.70 and $F(1,156)=9.31]$ and in the related-topics condition $[F(1,156)=61.35$ and $F(1,156)=10.76]$. In that the similarity condition $\times$ trials interaction was significant, an analysis of the simple main effects within each level of the repeated factor was conducted using a procedure recommended by Keppel (1973), in which individual error terms at the appropriate trial number were constructed. The results of this analysis revealed that the simple main effect was significant only at Trial $2[\mathrm{~F}(1,78)$ $=4.94]$; at Trial $1[\mathrm{~F}(1,78)<1]$ and Trial $3[\mathrm{~F}(1,78)$ $=2.96]$, the differences between conditions were not significant. An inspection of the individual error terms suggests that the main reason that the group differences were significant for Trial 2 but not for Trial 3 was that there was an increase in variability from Trial 2 to Trial 3 (MSe $=145.69$ vs. $\mathrm{MSe}=213.99$ ). Differences in group means were about the same on Trial 2 (mean $=5.92$ vs. 4.98) and Trial 3 (mean $=5.08$ vs. 4.08 ).

As in most studies of proactive interference, the vast majority of errors were errors of omission (failures to recall). By contrast, intrusions from previous trials, usually attributed to failures of list differentiation (Postman \& Keppel, 1977), tend to be rare. In the present study, it was sometimes difficult to ascertain with any degree of confidence whether errors were truly intrusions or were merely guesses. Accordingly, a fairly strict criterion for identifying prior-sentence intrusions was adopted. A prior-sentence intrusion was defined as the contiguous recall of two or more words that occurred consecutively or with only one other word intervening in an earlier sentence of the same block. Expressed as a percentage of the total number of errors, the total number of such intrusion errors was small, thus replicating the results of research with unconnected material. Also, there were no striking differences as a function of similarity condition or trial number (the percentages were between $1 \%$ and $2 \%$ ). However, the low frequency of intrusion errors does not mean that failures of list differentiation contributed little to the trials effect. The subjects may have withheld responses because they were uncertain about the sentence in which the responses occurred. The subjects may also have rejected implicit intrusions (which imply a failure of list differentiation) and have had insufficient time to retrieve the appropriate responses, even though the latter may have been available.

The final results to be reported were based on a repeated-measures analysis of Trial 3 versus Trial 4 . Traditionally, such an analysis provides evidence concerning release from proactive interference (Wickens, 1972). However, in the present experiment, Trial 4 sentences were used simply as buffer sentences to prevent the spread of interference from block to block, and in some respects they are not comparable to the sentences used on Trial 3 (e.g., most are concrete-a characteristic that is known to enhance recall-rather than abstract). Accordingly, no firm conclusions can be drawn concerning release. Nevertheless, the results of an analysis of Trial 3 versus Trial 4 could be enlightening. If, for example, Trial 4 performance was poorer than performance on Trial 3 , the con-

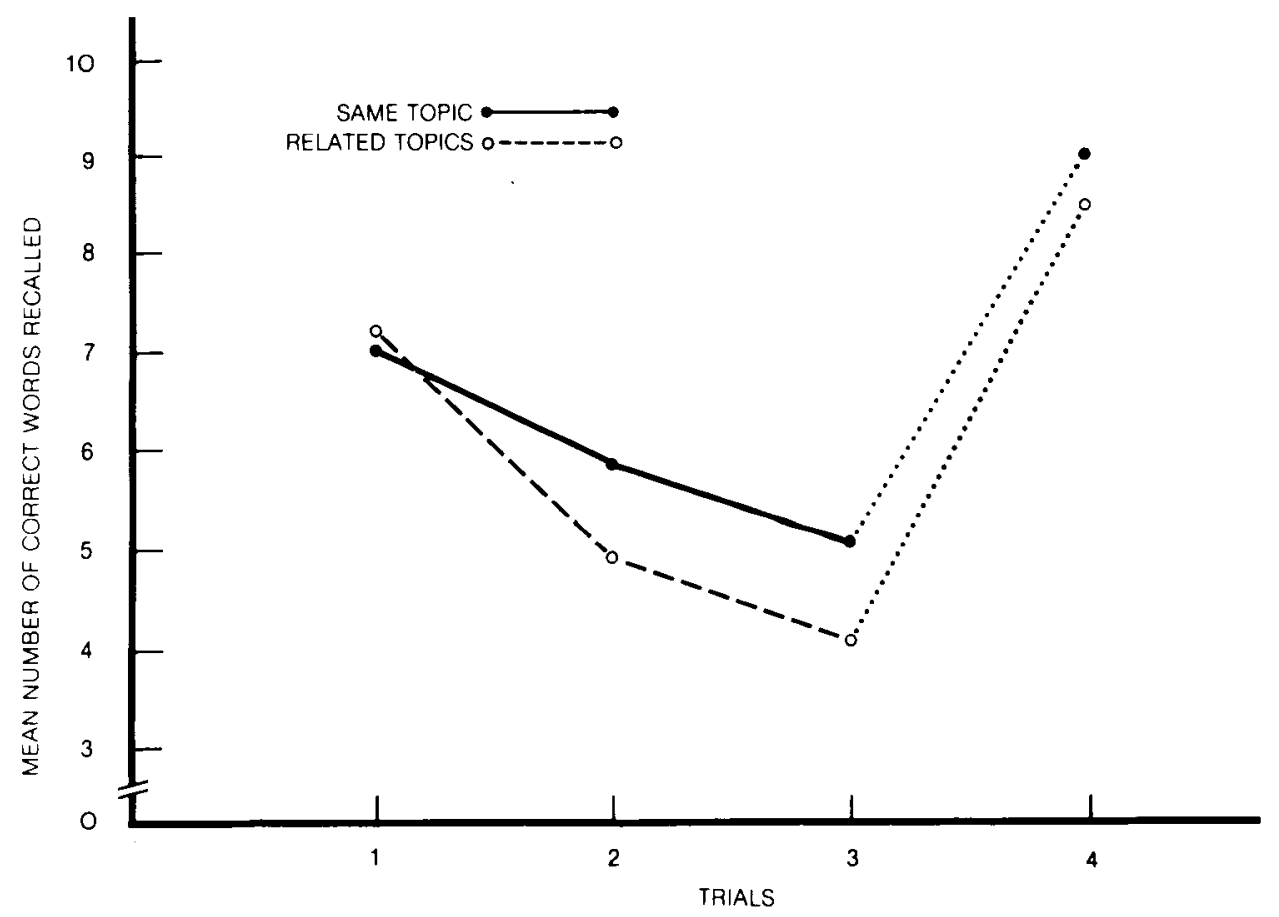

Figure 1. Mean number of correct words recalled as a function of similarity condition and trials (Experiment 1). 
clusions drawn from the Trial 1-3 analysis would have to be modified. The results of this analysis (same design as the primary analysis, but with one fewer trial) revealed a significant effect of trials $[F(1,78)=41.45]$; as expected, recall was considerably better on Trial 4 than on Trial 3. Neither the conditions effect $[F(1,78)=2.82]$ nor the more important conditions $\times$ trials interaction $[F(1,78)=1.39]$ was significant. Thus, recall on Trial 4 in both the same-topic condition (mean $=9.01$ ) and the related-topics condition (mean $=8.49$ ) was substantially superior to that on Trial 3 (see Figure 1).

Individual differences. Susceptibility to proactive interference was estimated using the formula (Trial 1 Trial 2)/Trial 2, in which the difference between Trials 1 and 2 is expressed as a proportion of Trial 1 recall. This procedure seemed to be more desirable than an absolutedifference measure because the latter would have confounded susceptibility to proactive interference with overall recall ability. Another important characteristic of this estimating procedure is that it does not take into account Trial 3 performance. This also seemed desirable because, in many instances, Trial 2 performance was so poor that there was virtually no recall to interfere with Trial 3 performance. Accordingly, in these instances Trial 3 could not be considered one in which there existed clear potential for interference.

All of the subjects contributing to interference estimates had been assigned to Order A. Thus, all subjects received identical materials on each trial. Efforts to obtain ACT scores for subjects in the Order B condition were discontinued for two reasons. First, most subjects in the study had not taken the ACT, and scores for those who did were frustratingly difficult to obtain. Second, for some inexplicable reason, a disproportionately large number of those who had not taken the ACT had been assigned to Order B. Accordingly, efforts to obtain ACT scores were eventually restricted to subjects assigned to Order A.

Correlations between susceptibility scores, scores on Trials 1 and 2, and ACT subtest scores are presented in Table 2. As can be seen, four of the eight correlations between susceptibility scores and subtest scores were significant, and all were negative: Subjects who had lower susceptibility scores tended to have higher ACT scores. In addition, three of the four significant correlations were obtained in the related-topics condition, the condition that was most sensitive to interference. Also of special note is the fact that the correlations between susceptibility scores and Trial 1 performance were weak and not significant. In addition, Trial 1 recall was correlated weakly with ACT scores in the related-topics condition. These data suggest that the correlations noted above were not due to something like general memory ability, but rather to susceptibility to interference per se. Finally, the fact that neither of the correlations between susceptibility scores and English Usage performance were significant suggests that achievement in academic areas stressing reading comprehension (the Mathematics Usage test contains some story problems) is more prone to interference than areas stressing punctuation, grammar, and sentence structure.

\section{EXPERIMENT 2}

The results of Experiment 1 indicated that successive sentences about the same topic and successive sentences about related topics were associated with substantial and progressive amounts of proactive interference; however, this effect was stronger in the related-topics condition. The question that arises, therefore, is what effect a succession of topically unrelated sentences would have on recall. Experiment 2 was designed with this question in mind.

\section{Method}

Subjects. The subjects were 40 students at the University of Nevada, Las Vegas, enrolled in undergraduate courses in educational psychology. They participated on a voluntary basis for extra course credit. None of the subjects had participated in Experiment 1.

Design, Materials, and Procedure. The design was mixed, with order (A, B) as a between-subjects factor and blocks (1-6) and trials $(1-4)$ as within-subject factors. Each subject was assigned randomly to the two levels of the order factor until there were 20 subjects in each. All aspects of the materials and procedures were identical to those in Experiment 1 (see Table 3), except that the sentences were about unrelated topics. Three sentences from each of the following six unrelated topics were constructed: heat, immigration, language, comedy, malaria, and law. The sentences were constructed by the experimenter on the basis of information found in an encyclopedia and a dictionary.

\section{Results and Discussion}

The same two scoring methods that had been used in Experiment 1 were applied to the raw data. The two were highly correlated $(\mathrm{r}=.94)$, and, as in Experiment 1, only

Table 2

Intercorrelations Among Selected Recall Scores and American College Test (ACT) Scores, Experiment 1 [Those from the Same-Topic Condition ( $=16$ ) Are Above the Diagonal, and Those From the Related-Topics Condition ( $\mathbf{N}=16)$ Are Below the Diagonal]

\begin{tabular}{lccccccc}
\multicolumn{1}{c}{ Scores } & 1 & 2 & 3 & 4 & 5 & 6 & 7 \\
\hline 1. Trial 1 $\left(\mathrm{T}_{1}\right)$ & 1.00 & $.82^{* *}$ & -.16 & $.62^{*}$ & .41 & .33 & $.63^{* *}$ \\
2. Trial 2 $\left(\mathrm{T}_{2}\right)$ & $.64^{* *}$ & 1.00 & $-.73^{* *}$ & $.62^{*}$ & .49 & .43 & $.73^{* *}$ \\
3. $\mathrm{T}_{1}-\mathrm{T}_{2} / \mathrm{T}_{1}$ & .08 & $-.71^{* *}$ & 1.00 & -.29 & -.19 & -.40 & $-.51^{*}$ \\
4. English Usage & .15 & .40 & -.32 & 1.00 & .23 & $.80^{* *}$ & $.60^{*}$ \\
5. Mathematics Usage & .29 & $.60^{*}$ & $-.51^{*}$ & .36 & 1.00 & .19 & $.72^{* *}$ \\
6. Social Science Reading & .23 & $.66^{* *}$ & $-.57^{*}$ & $.66^{* *}$ & .33 & 1.00 & $.55^{*}$ \\
7. Natural Science Reading & .32 & $.62^{*}$ & $-.50^{*}$ & $.77^{* *}$ & $.65^{* *}$ & .40 & 1.00 \\
\hline$* p<.05 . \quad * * p<.01$. & & & & & & &
\end{tabular}


Table 3

Design and Materials of Experiment 2 (Order A Is Shown; Order B Was Generated by Reversing the Order of Sentences 1-3 in Each Block)

\begin{tabular}{|c|c|c|}
\hline $\begin{array}{c}\text { Trial } \\
\text { Number }\end{array}$ & $\begin{array}{l}\text { Sentence } \\
\text { Notation }\end{array}$ & Actual Sentence \\
\hline & & Block 1 \\
\hline 1 & $\mathrm{Ht}_{1}$ & Heat is produced by alteration and conversion of the physical and chemical nature of various substances that store energy. \\
\hline 2 & $\operatorname{Im}_{2}$ & $\begin{array}{l}\text { Immigration is administered under laws that provide for the exclusion and deportation of aliens, particularly security } \\
\text { risks and subversives. }\end{array}$ \\
\hline 3 & $\operatorname{Lan}_{3}$ & $\begin{array}{l}\text { Language is a systematic means of communicating ideas or feelings by the use of conventionalized symbols having } \\
\text { understood meanings. }\end{array}$ \\
\hline \multirow[t]{2}{*}{4} & $\mathrm{C}_{1}$ & Clans consist of people who believe they descend from a common ancestor who lived in the distant mythological past. \\
\hline & & Block 2 \\
\hline 1 & $\mathrm{Co}_{1}$ & Comedy is a drama of a light and amusing nature, in which the weaknesses and manners of society are satirized. \\
\hline 2 & $\mathrm{Mal}_{2}$ & $\begin{array}{l}\text { Malaria results from a highly contagious, acutely noxious substance that once constituted a health problem of the first } \\
\text { magnitude. }\end{array}$ \\
\hline 3 & $\mathrm{Law}_{3}$ & $\begin{array}{l}\text { Law is a rule of conduct or action prescribed or formally recognized as enforceable by an acknowledged sovereign } \\
\text { authority. }\end{array}$ \\
\hline 4 & $\mathrm{G}_{1}$ & $\begin{array}{l}\text { Glass, even in its unstrengthened state, is an enormously useful material that is inexpensive, noncorrosive, and light } \\
\text { in weight. }\end{array}$ \\
\hline
\end{tabular}

Block 3
Immigration occurs in response to extreme scarcities caused by pestilence, blight, or unsettling changes in the prevail- ing climatic conditions.
Comedy develops through plots that emphasize the ludicrous and farcical incidents of life and the exasperated be- wilderment that follows.
Heat is released by a self-sustaining process during which carbon and hydrogen combine with oxygen in controlled amounts.
Antarctica is an ice-covered land surrounded by waters that house virtually untapped sources of marine protein and minerals.

\section{Block 4}

Language consists of words, their pronunciation, and the methods of combining them used and understood by a considerable community.

Law refers to a whole body of binding customs, practices, and rules that are advisable and obligatory to observe.

Malaria is characterized by unpleasant sensations of coldness, followed by malaise and high fever that eventually returns to normal.

Lightning is a natural spark, miles long, which usually accompanies a thunderstorm that developed from flat-bottomed cumulus clouds.

\section{Block 5}

Law is used as a means of regulating compliance to statutes, traditions, or generally accepted standards and redressing wrongs.

Heat develops rapidly under conditions of constant pressure, and is accompanied by the expansion of solids, gases, or vapors.

Immigration increases until the passing of free land and economic or social pressure lead to a movement for restriction.

Potatoes are vulnerable to many invading plant organisms, one of which caused a severe famine in nineteenth-century Ireland.

\section{Block 6}

Malaria develops rapidly in some cases and may result in extreme prostration, periodic attacks of delirium, and even unconsciousness.

Language refers to a formal system of signs, gestures, or marks, including rules for the transformation of admissable expressions.

Comedy is often ingeniously constructed and full of unraveling complicaitons, with the characters exemplifying entertaining and subtly varied emotions.

Mathematics, though often considered difficult and inapplicable, is useful in a vast range of commercial, technological, and professional occupations.

the results based on the verbatim method are reported.

Preliminary analysis (the alpha for all analyses was set at .05) indicated that neither order nor block was associated with systematic effects. Most importantly, there was no evidence of systematic differences in recall scores across successive blocks; the average recall scores for Blocks 1 through 6, respectively, were 5.22, 5.84, 5.27, $6.34,4.97$, and 4.72 . Therefore, subsequent analyses were collapsed over these factors.

Figure 2 presents the mean number of correct words 


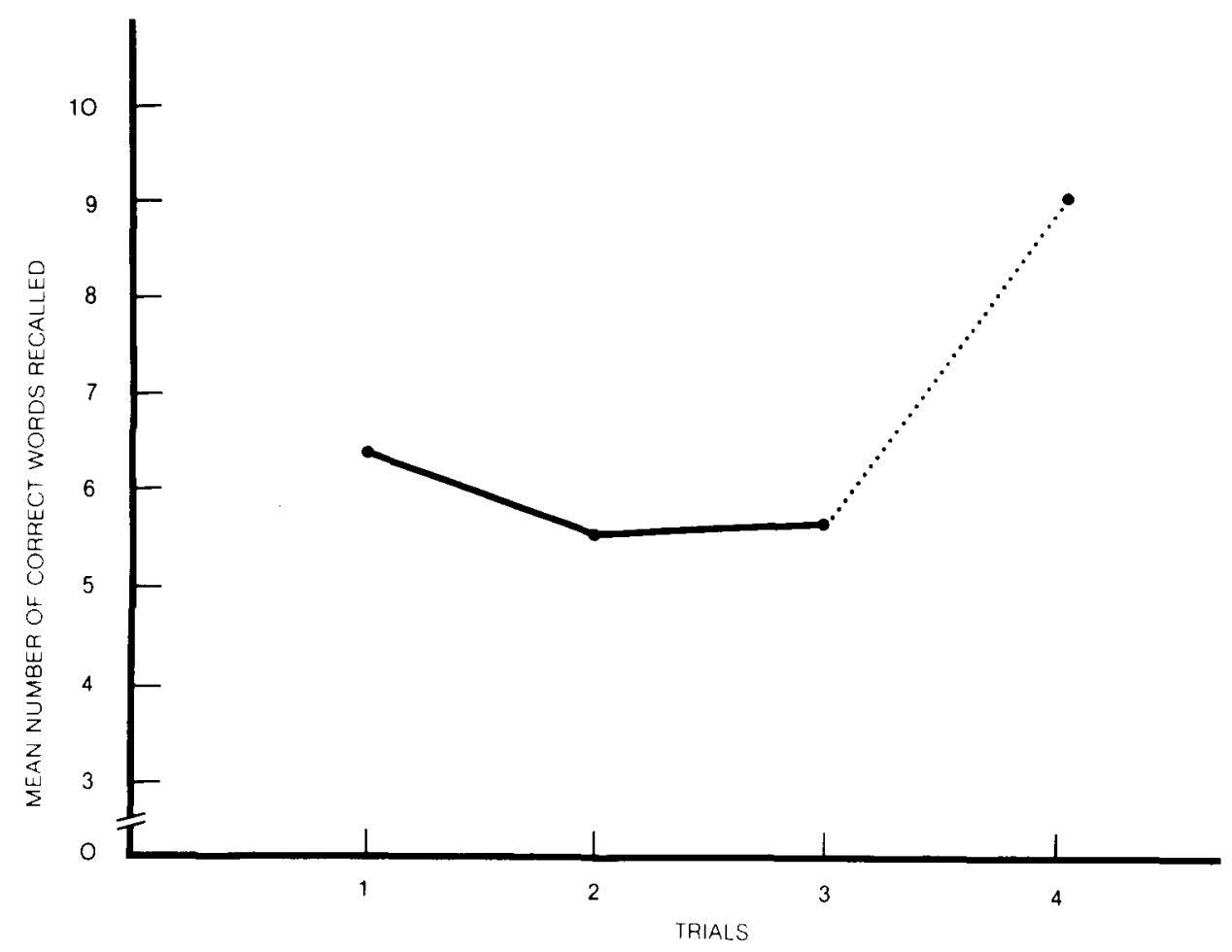

Figure 2. Mean number of correct words recalled as a function of trials (Experiment 2).

recalled as a function of trials. A repeated-measure ANOVA on these data revealed a significant trials effect $[F(2,78)=7.51]$. As can be seen, however, the nature of this effect was not the same as it had been in Experiment 1 . This impression was confirmed by simple contrasts between successive trials, which indicated that recall declined significantly only between Trials 1 and $2[F(1,78)$ $=10.32]$. By contrast, differences between Trial 2 and Trial 3 were negligible and not significant $[F(1,79)<1]$. Thus, a succession of sentences about unrelated topics was associated with some proactive interference; however, the effect was neither as strong nor as progressive as it had been in Experiment 1.

As in Experiment 1, there were relatively few priorsentence intrusion errors. Expressed as a percentage of total errors (well below 1\%), there were even fewer such intrusions than there were in the same-topic and the related-topics conditions.

Finally, a repeated-measures ANOVA of Trial 3 versus Trial 4 revealed a significant trials effect $[F(1,39)=$ 18.72]. This effect was attributable to the fact that the mean number of correct words recalled on Trial 4 was considerably greater than that recalled on Trial 3 (see Figure 2). Since there was relatively little proactive interference to be released from Trial 3, these results seem to confirm the expectation that Trial 4 sentences would prove to be less difficult, on average, than the others.

\section{GENERAL DISCUSSION}

The present findings indicate that short-term recall memory for sentences is susceptible to proactive inter- ference. In Experiment 1, substantial proactive interference was found under two conditions of topic similarity, but the effect was stronger when successively presented sentences were about related topics than when they were about the same topic. In addition, individual differences in susceptibility to proactive interference were significantly related to several tests of academic achievement. In Experiment 2, successively presented sentences about unrelated topics were also associated with proactive interference, but the effect was neither as large nor as progressive as it had been in Experiment 1.

Overall, these results add weight to the evidence that connected meaningful material is susceptible to proactive interference. In addition, they extend the results of previous studies by suggesting that some forms of topic organization are more susceptible to proactive interference than others, and that individual differences in susceptibility to proactive interference are a source of differences in academic achievement. Moreover, the two forms of topic organization used in this study are representative of the way lecture and text material is often organized. Successive sentences in a subdivision or paragraph are usually about the same topic, whereas sentences in adjacent subdivisions or paragraphs are often about related topics. Accordingly, the results of this study suggest that proactive interference may be a common casue of everyday learning problems, and are a first step in identifying the kinds of material that are most sensitive to proactive interference.

One finding that deserves particular attention is that of the impressive correlations between the susceptibility-toproactive-interference measure and ACT performance, es- 
pecially since they do not seem to be attributable to something like general memory ability. The sheer magnitude of these correlations is striking inasmuch as previous attempts to relate specific information-processing capabilities to standardized test scores have had only modest success and correlations above the .30s have rarely been reported (see Hunt, 1980). Accordingly, this finding suggests that differences in susceptibility to proactive interference may be an important source of differences in scholastic achievement.

The present results also raise a theoretical question: that of accounting for the effects of topic similarity. Recent data (J. R. Anderson, 1983; Wickens, Moody, \& Dow, 1981) suggest that cumulative proactive interference is caused largely by the disruption of retrieval by alternative response sets (Newton \& Wickens, 1956; Postman, Stark \& Fraser, 1968). This disruption is assumed to occur during the initial act of the memory process, which is an attempt to retrieve the entire memory set. Furthermore, this process seems to be hampered if other similar, but not identical, memory sets have been stored. However, the many differences between the materials used here, and traditional ones upon which this account is based, prevent it from providing an easy explanation of the present findings. One important difference is that meaningful sentences were used here: Each "list" consisted of a sentence rather than of a collection of unconnected material. In addition, each sentence began with a topic word that might be expected to function as a list marker. In view of these differences, it is unclear what predictions the response-set hypothesis would make. On the one hand, the fact that the topic word in the sametopic condition was identical for three successive trials seems to argue for maximum interference in the relatedtopics condition, as was found. On the other hand, since each individual sentence contained many different key words, more interference should have been expected in the most similar, but not identical, same-topic condition. This difficulty is complicated by the fact that the topic word was recalled more than $90 \%$ of the time in all similarity conditions. Thus, the subjects rarely were unable to retrieve the list markers, in contrast to what might be expected on the basis of response-set interference. In short, this hypothesis, as traditionally formulated, does not provide an adequate account of the present findings.

With a few additional assumptions, however, it seems possible to retain the flavor of the response-set hypothesis and to use it to account for the present results. To begin with, it can be assumed that once the topic word is assessed, disruption occurs in the process of "unpacking" the sentence, since each sentence, like a list of words, has a semantic character all its own. Second, it may be assumed that disruption occurs as a result of the automatic subthreshold activation, or priming of other neighboring concepts, which would make them more accessible than concepts in their latent state. The assumption that activation has an incremental effect on the accessibility of a con- cept is clearly not unprecedented (e.g., James, 1890; J. R. Anderson, 1983), and there are data that favor a distinction between items that are in a recently retrieved state and those in a latent state (Dosher, 1981). With these assumptions in place, the effect of topic similarity on recall may be understood, although precise predictions still cannot be made.

Consider the sentence blocks in the three similarity conditions used in the present study (see Tables 1 and 3). In the same-topic condition, successive sentences (Trials 1 3 ) tend to share several of the same or similar concepts. For example, in Block 1, Sentence $A_{1}$ refers to "people who feel inadequate and fearful," Sentence $A_{2}$ refers to "people under stress and indecisive as a result of numerous academic demands," and Sentence $A_{3}$ refers to "people who fear embarrassment or failure." In Block 2, Sentence $\mathrm{H}_{1}$ characterized hostility as "harmful, destructive, and alienating," Sentence $\mathrm{H}_{2}$ refers to hostility "expressed in angry, prejudiced, and biased arguments," and Sentence $\mathrm{H}_{3}$ refers to hostility in terms of "dominance, excessive criticism, and little tolerance for others." By contrast, successive sentences in the related-topics condition share relatively few concepts, and successive sentences in the unrelated-topics condition tend to express very dissimilar concepts.

The priming and activation of sentence concepts in each of the conditions is illustrated in Figure 3 with sentences from Trials 1 and 2, Block 1, Order A. Note that in the same-topic condition, the activation of the concepts expressed by the words "under stress and indecisive as a result of numerous academic demands" (stress ... demands) primes a related concept "fear," which, in turn, primes "anxiety," the topic concept of Sentences $A_{1}$ and $A_{2}$. But, whereas some of the concepts of Sentence $A_{1}$ may be reactivated and primed (resulting in some interference), the activation of relevant Sentence $A_{2}$ concepts is likely to continue. By contrast, the priming of "anxiety" in the related-topics condition, which follows from the activation of "avoids simple challenges" (avoids ... challenges) and the priming of the related concept of "fear," is likely to reactivate and prime only Sentence $A_{1}$ concepts, resulting in greater amounts of proactive interference. This process, by which there is massive arousal of inappropriate concepts, may underlie response-set interference. Finally, priming in the unrelated-topics condition should not result in nearly as much priming and reactivation of Sentence 1 concepts. Thus, large amounts of proactive interference should not occur.

This account suggests some interesting possible loci of individual differences in susceptibility to proactive interference. One possibility is in the priming and activation processes: Individuals who are relatively susceptible to proactive interference may simply have a tendency to prime and activate concepts less discriminatingly than those who are relatively insensitive to proactive interference, resulting in the reactivation of task-irrelevant concepts. A second possibility is in the time course of priming 
SAME TOPIC

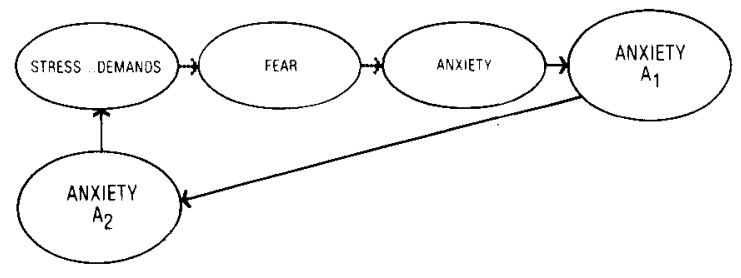

RELATED TOPICS

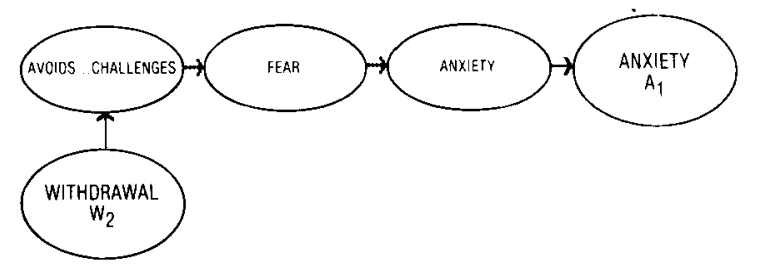

UNRELATED TOPICS

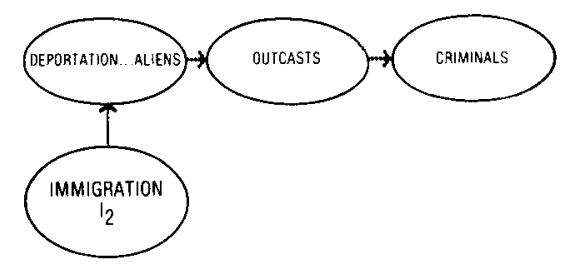

Figure 3. The activation (solid lines) and priming (broken lines) of representative fragments of Trial 1 and Trial 2 sentences.

and activation processes. For some individuals, activated concepts may remain in a highly accessible state for a longer period of time than they do for others. Thus, the potentially interfering effects of concepts activated earlier may persist for a longer period of time. One goal of future research will be to explore these and other possibilities.

In closing, a few final comments are in order. A critic might charge that the present materials oriented the subjects primarily to word-for-word or rote memorization, thus stripping the sentences of their meanings. There are several reasons for believing that this was not the case. First, R. C. Anderson (1971) found that, following the presentation of sentences such as "The uncle shouted an obscene remark," later recall of the subject term ("uncle") was cued almost as well by a paraphrase of the rest of the sentence ("yelled some dirty word") as by the originally presented words. Moreover, this result was also found for subjects instructed to learn the original sentences by "rote memorization." The implication is that even with instructions designed to foster "nonnormal" rote processing of sentences, subjects nonetheless establish meaningful associations (see also Bower, 1974). Second, whereas the present sentences were more complex than those used by $\mathbf{R}$. C. Anderson, there were a substantial number of paraphrases and synonymous substitutions. This would not be expected if subjects were simply rote processing the sentences.

In a similar vein, the critic might charge that a distractor task, used here to minimize recall from short-term memory, has no ecological validity. Although it is true that distractor tasks per se are not used in the classroom and other real-life situations, it seems obvious that people are continually distracted by the demands imposed by the steady flow of information inherent to normal listening. As a consequence, subsequent recall depends entirely, or almost entirely, on retrieval from long-term memory. In effect, then, the distractor paradigm is probably a better analogue of normal listening than is a paradigm in which the subject recalls immediately. In short, the present results, although collected using a traditional verbal learning procedure, raise some serious questions about the role of proactive interference in everyday learning.

\section{REFERENCES}

ANDERSON, J. R. (1983). A spreading activation theory of memory. Journal of Verbal Learning and Verbal Behavior, 22, 261-295.

ANDERSON, R. C. (1971). Encoding processes in the storage and retrieval of sentences. Journal of Experimental Psychology, 91, 338-340.

Blumenthal, G. B., \& Robbins, D. (1977). Delayed release from proactive interference with meaningful material: How much do we remember after reading brief prose passages? Journal of Experimental Psychology: Human Learning and Memory, 3, 754-761.

BowER, G. H. (1974). Selective facilitation and interference in retention of prose. Journal of Educational Psychology, 66, 1-8.

DOSHER, B. A. (1981). The effects of delay and interference: A speedaccuracy study. Cognitive Psychology, 13, 551-582.

Gunter, B., Berry, C., \& Clifford, B. R. (1981). Proactive interference effects with television news items: Further evidence. Journal of Experimental Psychology: Human Learning and Memory, 7, 480-487.

Gunter, B., Clifford, B. R., \& BerRy, C. (1980). Release from proactive interference with television news items: Evidence for encoding dimensions within televised news. Journal of Experimental Psychology: Human Learning and Memory, 6, 216-223.

Hopkins, R. H., Edwards, R. E., \& CoOK, C. L. (1972). The dissipation and release of proactive interference in a short-term memory task. Psychonomic Science, 27, 65-67.

HUNT, E. (1980). Intelligence as an information-processing concept. British Journal of Psychology, 71, 449-474.

JAMES, W. (1890). The principles of psychology. New York: Holt, Rinehart \& Winston.

KEPPEL, G. (1973). Design and analysis: A researcher's handbook. Englewood Cliffs, NJ: Prentice-Hall.

LoEss, H., \& WAUGH, N. C. (1967). Short-term memory and inter-trial interval. Journal of Verbal Learning and Verbal Behavior, 6, 455-460.

Newton, J. M., \& WiCKens, D. D. (1956). Retroactive inhibition as a function of the temporal position of interpolated learning. Journal of Experimental Psychology, 51, 149-154.

PostMAN, L., \& KEPPEL, G. (1977). Conditions of cumulative proactive inhibition. Journal of Experimental Psychology: General, 106, 376-403.

Postman, L., Stark, K., \& Fraser, J. (1968). Temporal changes in interference. Journal of Verbal Learning and Verbal Behavior,7, 672-694.

ThORNDYKE, P. W. (1977). Cognitive structures in comprehension and memory of narrative discourse. Cognitive Psychology, 9, 77-110.

ThoRNDYKe, P.W., \& HAYEs-Roth, B. (1979). The use of schemata in the acquisition and transfer of knowledge. Cognitive Psychology, 11, 82-106.

Underwood, B. J. (1957). Interference and forgetting. Psychological Review, 64, 49-60.

WickeNs, D. D. (1972). Characteristics of word encoding. In A. W. Melton \& E. Martin (Eds.), Coding processes in human memory. Washington, DC: Winston.

Wickens, D. D., MoODY, M. J., \& Dow, R. (1981). The nature and timing of the retrieval process and of interference effects. Journal of Experimental Psychology: General, 110, 1-20.

(Manuscript received May 13, 1984; revision accepted for publication November 7,1984 .) 